\title{
Impact of dust cooling on direct collapse black hole formation
}

\author{
M. A. Latif ${ }^{1,2}$, K. Omukai ${ }^{3}$, M. Habouzit ${ }^{1,2}$, D. R. G. Schleicher ${ }^{4}$, M. Volonteri ${ }^{1,2}$ \\ Sorbonne Universits, UPMC Univ Paris 06, UMR 7095, Institut d'Astrophysique de Paris, F-75014, Paris, France \\ CNRS, UMR 7095, Institut d'Astrophysique de Paris, F-75014, Paris, France \\ Astronomical Institute, Tohoku University, Aoba, Sendai 980-8578, Japan \\ Departamento de Astronoma, Facultad Ciencias Fsicas y Matemticas, Universidad de Concepcin, \\ Av. Esteban Iturra s/n Barrio Universitario, Casilla 160-C, Chile
}

latif@iap.fr

\begin{abstract}
Observations of quasars at $z>6$ suggest the presence of black holes with a few times $10^{9} \mathrm{M}_{\odot}$. Numerous models have been proposed to explain their existence including the direct collapse which provides massive seeds of $10^{5} \mathrm{M}_{\odot}$. The isothermal direct collapse requires a strong Lyman-Werner flux to quench $\mathrm{H}_{2}$ formation in massive primordial halos. In this study, we explore the impact of trace amounts of metals and dust enrichment. We perform three dimensional cosmological simulations for two halos of $>10^{7} \mathrm{M}_{\odot}$ with $\mathrm{Z} / \mathrm{Z}_{\odot}=10^{-4}-10^{-6}$ illuminated by an intense Lyman Werner flux of $\mathrm{J}_{21}=10^{5}$. Our results show that initially the collapse proceeds isothermally with $\mathrm{T} \sim 8000 \mathrm{~K}$ but dust cooling becomes effective at densities of $10^{8}-10^{12} \mathrm{~cm}^{-3}$ and brings the gas temperature down to a few $100-1000 \mathrm{~K}$ for $\mathrm{Z} / \mathrm{Z}_{\odot} \geq 10^{-6}$. No gravitationally bound clumps are found in $\mathrm{Z} / \mathrm{Z}_{\odot} \leq 10^{-5}$ cases by the end of our simulations in contrast to the case with $\mathrm{Z} / \mathrm{Z}_{\odot}=10^{-4}$. Large inflow rates of $\geq 0.1 \mathrm{M}_{\odot} / \mathrm{yr}$ are observed for $\mathrm{Z} / \mathrm{Z}_{\odot} \leq 10^{-5}$ similar to a zero-metallicity case while for $\mathrm{Z} / \mathrm{Z}_{\odot}=10^{-4}$ the inflow rate starts to decline earlier due to the dust cooling and fragmentation. For given large inflow rates a central star of $\sim 10^{4} \mathrm{M}_{\odot}$ may form for $\mathrm{Z} / \mathrm{Z}_{\odot} \leq 10^{-5}$.
\end{abstract}

Subject headings: methods: numerical - cosmology: theory - early Universe - galaxies: formation

\section{Introduction}

The discovery of high redshift quasars at $z>6$ reveals the presence of supermassive black holes (SMBHs) of about a few billion solar masses (Fan et al. 2006; Willott et al. 2010; Mortlock et al.2011; Venemans et al 2013; Wu et al. 2015; Venemans et al. 2015). The formation of SMBHs a few hundred million years after the Big Bang presents a challenge for our understanding of early structure formation. The pathways for their assembly include the stellar mass black holes forming from the collapse of Pop III stars (Abel et al. 2002; Yoshida et al. 2008; Latif et al. 2013c; Hirano et al. 2014), black hole seeds from the collapse of a dense nuclear cluster either due to the relativistic instability Baumgarte \& Shapiro 1999), via stellar dynamical process (Omukai et al. 2008; Devecchi \& Volonteri 2009; Katz et al. 2015; Yajima \& Khochfar 2015), even the core collapse of a cluster consisting of stellar mass black holes
(Davies et al. 2011; Lupi et al. 2014) or a direct collapse of a protogalactic gas cloud (Loeb \& Rasio 1994; Bromm \& Loeb 2003; Begelman et al. 2006; Spaans \& Silk 2006; Latif et al. 2013a. d; Ferrara et al. 2014: Inayoshi et al. 2014; Choi et al. 2015; Shlosman et al. 2016). Details of these models are discussed in dedicated reviews (Volonteri 2010; Volonteri \& Bellovary 2012; Haiman 2012).

The masses of seed black holes from the above mentioned scenarios range from a few hundred to thousand solar masses except the direct collapse model which provides massive seeds of about $10^{5}-10^{6} \mathrm{M}_{\odot}$. The lighter seeds require continuous accretion close to the Eddington limit in order to reach a billion solar masses in a few hundred million years. Therefore, massive seeds forming via the direct collapse are favoured for the assembly of high redshift quasars. The formation of so-called direct collapse black holes (DCBHs) requires large inflow rates of about 
$\geq 0.1 \mathrm{M}_{\odot} / \mathrm{yr}$ (Begelman 2010; Hosokawa et al. 2013; Schleicher et al. 2013; Sakurai et al. 2015). These inflow rates can be obtained either via dynamical processes such as the 'bars-in-bars' instability (see Begelman et al. (2006) and Begelman \& Shlosman (2009)) or thermodynamically due to a large speed of sound $\left(\dot{\mathrm{M}} \sim \mathrm{c}_{\mathrm{s}}^{3} / \mathrm{G} \sim 0.1 \mathrm{M}_{\odot} / \mathrm{yr}(\mathrm{T} / 8000 \mathrm{~K})^{3 / 2}\right.$, where $c_{s}$ is the thermal sound speed). Large inflow rates can be more easily achieved in an isothermal direct collapse which necessitates the presence of a strong Lyman Werner (LW) flux to quench the formation of molecular hydrogen in metal free halos (Omukai 2001; Schleicher et al. 2010; Shang et al. 2010; Latif et al. 2014). The critical value of the LW flux $\left(\mathrm{J}_{\text {crit }}\right)$ above which isothermal collapse occurs depends on the spectra of the stars (Omukai 2001; Shang et al. 2010; Latif et al. 2014; Sugimura et al. 2014; Agarwal \& Khochfar 2015; Regan et al. 2014b; Latif et al. 2015). Latif et al. (2015) computed the strength of $\mathbf{J}_{\text {crit }}$ for realistic Pop II spectra from three dimensional cosmological simulations and found that it corresponds to a value of $\mathrm{J}_{21}$ of a few times $10^{4}$ where $\mathrm{J}_{21}=1 \mathrm{implies}$ a flux of $10^{-21} \mathrm{erg} / \mathrm{cm}^{2} / \mathrm{s} / \mathrm{Hz} / \mathrm{sr}$ below the Lyman limit. Another constraint for the isothermal direct collapse is that the halos should be metal free as trace amounts of metals and dust can lead to fragmentation and star formation (Omukai et al. 2008).

Numerical simulations show that large inflow rates of about $0.1-1 \mathrm{M}_{\odot} / \mathrm{yr}$ are available in the massive primordial halos of $10^{7}-10^{8} \mathrm{M}_{\odot}$ illuminated by a strong LW flux at $\mathrm{z}=15$ and massive objects of $10^{5} \mathrm{M}_{\odot}$ can be formed within about 100,000 years (Wise et al. 2008; Latif et al. 2013a d; Regan et al. 2014a; Becerra et al. 2015; Inayoshi et al. 2014; Van Borm et al. 2014). However, the value of $\mathbf{J}_{c r i t}\left(>10^{4}\right.$ in terms of $\left.\mathbf{J}_{21}\right)$ required for the formation of isothermal DCBHs is much higher than the background UV flux and such a flux can only be achieved in the close vicinities of star forming galaxies (Diikstra et al. 2008; Agarwal et al. 2012; Diikstra et al. 2014; Visbal et al. 2014; Latif et al. 2015; Habouzit et al. 2015). Supernova winds from nearby galaxies can also pollute halos with metals and make the sites for an isothermal direct collapse even more rare (Johnson et al. 2013). In the context of second generation star formation (Pop II), it has been found that above a critical value of metallically $\left(Z_{\text {crit }}=3 \times 10^{-4} Z_{\odot}\right)$ fragmentation becomes inevitable and leads to the formation of Pop II stars (Omukai et al. 2005; Glover \& Jappsen
2007; Wise et al. 2012; Safranek-Shrader et al. 2014; Bovino et al. 2014; Ritter et al. 2015). The critical value of the metallicity is further reduced by about two orders of magnitude in the presence of trace amounts of dust Schneider et al. 2003; Omukai et al. 2005, 2008) and the $\mathrm{H}_{2}$ formation on dust grains enhances the cooling (Omukai 2001; Cazaux \& Spaans 2009; Latif et al. 2012). Numerical simulations show that low mass star formation seems plausible due to the dust cooling occurring at high densities in minihalos with metallicities as low as a few times $10^{-5}$ solar (Tsuribe \& Omukai 2006, 2008; Dopcke et al. 2013; Smith et al. 2015).

Omukai et al. (2008) explored the thermodynamical properties of gas irradiated by a strong LW flux above $J_{\text {crit }}$ and enriched by trace amounts of metals and dust. They found from a one-zone model that in such conditions dust cooling becomes effective for $\mathrm{Z} / \mathrm{Z}_{\odot} \geq 5 \times 10^{-6}$ and proposed that a dense stellar cluster can form under these conditions which may later collapse into a black hole of $100-1000 \mathrm{M}_{\odot}$.

In this study, we explore the impact of dust and metal line cooling in massive primordial halos illuminated by a strong background LW flux and polluted by trace amounts of metals and dust. To accomplish this goal, we perform high resolution three dimensional cosmological simulations for two halos of a few times $10^{7} \mathrm{M}_{\odot}$ by turning on a LW flux of strength $10^{5}$ in units of $J_{21}$ at $\mathrm{Z}=30$ and presume that they are pre-enriched with $\mathrm{Z} / \mathrm{Z}_{\odot}=10^{-6}-10^{-4}$. Our results suggest that dust cooling occurs even for $\mathrm{Z} / \mathrm{Z}_{\odot}=10^{-6}$ at densities above $10^{10} \mathrm{~cm}^{-3}$ but still the conditions are suitable for the formation of massive objects. This work has important implications for assessing the feasibility of black hole formation models.

This article is organised as follows. In section 2, we describe numerical methods and chemical model employed in this work. We present our results in section 3 and discuss its implications for black hole formation in section 4. Summary of our main results and conclusions are discussed in section 5 .

\section{Numerical Methods}

We have performed simulations using the open source code Enzo version 2.4 (Bryan et al. 2014). Enzo is an adaptive mesh refinement, parallel, grid based cosmological simulation code which has been extensively used to perform high resolution simulations. It uses the message passing interface (MPI) 
to achieve scalability and portability on various platforms. The piece-wise parabolic method (PPM) is employed to solve hydrodynamics, the particle-mesh technique (PM) is used to solve dark matter dynamics and the multi-grid Poisson solver for gravity.

\subsection{Simulation setup}

Our simulations are started with cosmological initial conditions at $\mathrm{z}=100$ generated with the "inits" package available with Enzo. The computational domain has a size of $1 \mathrm{Mpc} / \mathrm{h}$ in comoving units and periodic boundary conditions are adopted both for gravity and hydrodynamics. We use parameters from the WMAP 7 years data to generate initial conditions (Jarosik et al. 2011). Our computational box is centered on the most massive halo forming at $\mathrm{z}=15$ selected from uniform grid DM only simulations of resolution $128^{3}$ particles. We rerun the simulations with a top grid resolution of $128^{3}$ grid cells (same number of DM particles) and add two nested refinement levels each with a resolution of $128^{3}$ grid cells and $128^{3}$ DM particles. In all, we employ 5767168 particles to solve the DM dynamics which provide us an effective DM resolution of about $600 \mathrm{M}_{\odot}$. We smooth DM particles at about a 2 pc scale to avoid spurious numerical artefacts, see Latif \& Volonteri (2015) for a detailed discussion. We further add 28 dynamical refinement levels during the course of the simulations in the central $62 \mathrm{kpc}$ comoving region of the box. This approach enables us to resolve the gravitational collapse down to scales of about 0.1 AU. We employ a fixed Jeans resolution of 32 cells throughout the simulations to resolve turbulent eddies and to fulfil the Truelove criterion which requires a resolution of at-least four cells per Jeans length (Truelove et al. 1997; Federrath et al. 2011; Latif et al. 2013b). Our dynamical refinement criterion further includes the baryonic over density and particle mass resolution. Grid cells are marked for refinement if gas density exceeds four times the cosmic mean. Similarly, cells are flagged for refinement if DM density is above 0.0625 times $\rho_{D M} r^{\ell \alpha}$ where $\rho_{D M}$ is the dark matter density, $\mathrm{r}=2$ is the refinement factor, $\ell$ is the refinement level, and $\alpha=-0.3$ makes the refinement super-Lagrangian.

We select two halos of $5.6 \times 10^{7} \mathrm{M}_{\odot}$ and $3.25 \times$ $10^{7} \mathrm{M}_{\odot}$. The properties of these halos such as their spins, collapse redshifts and $\mathrm{J}_{\text {crit }}$ are listed in table 1 of Latif \& Volonteri (2015) as the halo A and the halo C, respectively. We presume that the halos are enriched with trace amount of metals and dust by supernova winds from a nearby star forming galaxy. The latter also provides a strong LW flux above the critical threshold required for the quenching of $\mathrm{H}_{2}$ formation. The prime aim of this study is to explore the impact of dust cooling in the presence of an intense LW flux. We therefore select $\mathbf{J}_{21}=10^{5}$ for which halo collapses isothermally in the absence of dust cooling. At densities above $10^{-16} \mathrm{~g} / \mathrm{cm}^{3}$ where dust cooling becomes important the gas cloud is already self-shielded against external radiation and therefore the value of $\mathrm{J}_{\text {crit }}$ does not depend on the metal content of the halo. This scenario is particularly relevant for the halos which get polluted while being irradiated by a strong LW flux, and we specifically explore here the cases with $\mathrm{Z} / \mathrm{Z}_{\odot}=10^{-6}, 10^{-5}$ and $10^{-4}$.

Due to the computational constraints, we stop our simulations when a peak density of $3 \times 10^{-11} \mathrm{~g} / \mathrm{cm}^{3}$ is reached. Omukai et al. (2008) have shown that dust cooling becomes optically thick at densities above $\sim 10^{-10} \mathrm{~g} / \mathrm{cm}^{3}$ for the metallicities explored here, and thereafter collapse is expected to proceed adiabatically. The transition to the adiabatic regime during the gravitational collapse of primordial gas has been explored in hydrodynamical simulations (Inayoshi et al. 2014; Van Borm et al. 2014; Becerra et al. 2015; Latif et al. 2016). These studies found that the adiabatic phase stabilises the gravitational collapse on small scales, breaks its selfsimilarity and a protostar begins to form at this stage. For dust cooling, Omukai et al. (2008) have shown that the transition to the adiabatic phase occurs at earlier stages of the collapse (at lower densities compared to the primordial gas). They also considered the sputtering of dust grains in their model finding that the dust temperature remains below the vaporisation temperature in optically thin regime and once adiabatic phase begins, it exceeds the evaporation temperature but gas cloud is already optically thick. Therefore, thermal evolution remains adiabatic similar to the metal free gas. Further details of our model are described in Omukai et al. (2008).

In this study, we explore three cases with metallicities of $\mathrm{Z} / \mathrm{Z}_{\odot}=10^{-6}, 10^{-5}$ and $10^{-4}$ for each halo and turn on a strong LW flux of strength $\mathrm{J}_{21}=10^{5}$, well above the $\mathrm{J}_{\text {crit }}$ found in Latif et al. (2015). Further details of our chemical network as well as heating and cooling processes are described in the section below. 


\subsection{Chemical model}

We employ the KROME package Grassi et al. 2014) to solve the chemical and thermal evolution of the gas in cosmological simulations. The rate equations of $\mathrm{H}, \mathrm{H}^{+}, \mathrm{H}^{-}, \mathrm{He}, \mathrm{He}^{+}, \mathrm{He}^{++}, \mathrm{H}_{2}, \mathrm{H}_{2}^{+}, \mathrm{e}^{-}$are solved to study their evolution during the gravitational collapse. We employ a uniform background LW flux of strength $\mathrm{J}_{21}=10^{5}$ emitted from a Pop II galaxy which can be mimicked by a radiation temperature of $2 \times 10^{4} \mathrm{~K}$ (see Sugimura et al. (2014); Latif et al. (2015)). Our results do not depend on the choice of the radiation spectrum as long as the flux is well above the critical threshold to dissociate molecular hydrogen (derived by Omukai et al. (2008); Sugimura et al. (2014) for the one-zone model and by Latif et al. (2015) in 3D simulations). The list of chemical reactions is given in table 1 in the appendix of Latif et al. (2015). Our model includes $\mathrm{H}_{2}$ formation, $\mathrm{H}_{2}$ and $\mathrm{H}_{2}^{+}$ photo-dissociation, $\mathrm{H}^{-}$photo-detachment, collisional induced emission and $\mathrm{H}_{2}$ collisional dissociation. In addition to this, we add a reaction for $\mathrm{H}_{2}$ formation on dust grain surfaces taken from Omukai (2000). Various cooling and heating mechanisms such as cooling due to the collisional excitation, collisional ionisation, radiative recombination, Bremsstrahlung radiation and chemical cooling/heating from three-body reactions are included in the chemical model. We also employ the $\mathrm{H}_{2}$ self-shielding fitting formula given in Wolcott-Green et al. (2011).

In addition to the cooling and heating processes mentioned above, we include cooling due to the fine structure lines of CII and OI, cooling and heating by dust grains which is relevant for $\mathrm{Z} / \mathrm{Z}_{\odot}=$ $10^{-6}, 10^{-5}$ and $10^{-4}$. Our treatment for metal and dust cooling/heating processes comes from the model of Omukai et al. (2008), here we summarise their main features. We do not explicitly include the chemical reactions involving metals but instead assume that most of the oxygen and carbon are in OI and CII form due to the presence of a strong LW flux. Carbon has a lower ionisation energy $(11.26 \mathrm{eV})$ than hydrogen and therefore is in CII form while OI remains in the atomic state due to the charge exchange with hydrogen atoms in a neutral medium. The dust grain composition and size distribution is assumed to be similar to the solar neighbourhood and the amount is scaled with the metallicity of the gas cloud. We assume a dust to gas mass ratio of $0.01 \mathrm{Z} / \mathrm{Z}_{\odot}$. Dust grains in the supernova ejecta are more efficient in cooling and $\mathrm{H}_{2}$ formation due to their smaller size and larger surface area
(Schneider et al. 2003; Omukai et al. 2008). The dust is assumed to be in thermal equilibrium and its temperature $\left(\mathrm{T}_{\text {dust }}\right)$ is evaluated separately from the gas temperature at each density and temperature. The following energy balance equation is solved to compute the $\mathrm{T}_{\text {dust }}$ Omukai et al. 2008)

$$
\begin{array}{r}
4 \pi \int \kappa_{a, v} B_{v}\left(T_{d u s t}\right) d v=\Lambda_{g a s \rightarrow d u s t} \\
+4 \pi \int \kappa_{a, v} B_{v}\left(T_{C M B}\right) d v
\end{array}
$$

Here $\Lambda_{\text {gas } \rightarrow \text { dust }}$ is the net gas cooling rate per unit mass due to dust grain collisions, $\kappa_{a, v}$ is the dust absorption opacity and $B_{v}$ is the black body spectrum. $\Lambda_{\text {gas } \rightarrow \text { dust }}$ is given as (Hollenbach \& McKee 1979)

$$
\begin{gathered}
\Lambda_{\text {gas } \rightarrow \text { dust }}=5.8 \times 10^{-8} n\left(\frac{T}{1000}\right)^{1 / 2} \times \\
{\left[1-0.8 \exp \left(\frac{-75 K}{T}\right)\right]\left(T-T_{\text {dust }}\right)\left(Z / Z_{\odot}\right)}
\end{gathered}
$$

where $\mathrm{n}$ is the gas number density and $\mathrm{T}_{\text {dust }}$ is the dust temperature. We have set temperature floor at $\mathrm{T}_{\mathrm{CMB}}$. Further details about the dust properties can be found in section 2.2 of Omukai et al. (2008).

\section{Results}

We here present our main results obtained both from a one-zone model and three dimensional cosmological simulations.

\subsection{One-zone model}

To test the chemical model presented in the previous section, we performed a one-zone test with a LW flux of strength $\mathrm{J}_{21}=10^{5}$ (well above the $\mathrm{J}_{\text {crit }}$ from one-zone model with $\mathrm{T}_{\mathrm{rad}}=2 \times 10^{4} \mathrm{~K}$ ) and varied the metallicity from $\mathrm{Z} / \mathrm{Z}_{\odot}=10^{-6}-10^{-4}$. We took an initial temperature of $160 \mathrm{~K}$, a gas density of $\sim 10^{-23} \mathrm{~g} / \mathrm{cm}^{3}$ and species abundances of $2 \times 10^{-6}$ for $\mathrm{e}^{-}$and $2 \times 10^{-4}$ for $\mathrm{H}_{2}$. In Figure 1 we show the temperature and the abundances of $\mathrm{H}_{2}, \mathrm{H}^{-}$and $\mathrm{e}^{-}$as a function of density. In the presence of a strong LW flux, $\mathrm{H}_{2}$ gets photo-dissociated, its abundance remains below $10^{-8}$ and is unable to cool the gas. The temperature increases up to about $10^{4} \mathrm{~K}$ where atomic line cooling becomes effective and cools the gas down to $8000 \mathrm{~K}$ up to the densities of $10^{-17} \mathrm{~g} / \mathrm{cm}^{3}$. At higher densities for $\mathrm{Z} / \mathrm{Z}_{\odot} \geq 10^{-5}$, dust cooling comes into play and sharply cools the gas down to a few hundred 
$\mathrm{K}$. For the case of $\mathrm{Z} / \mathrm{Z}_{\odot}=10^{-4}, \mathrm{H}_{2}$ formation on dust grains becomes effective and its abundances get boosted very quickly. The small increase in the temperature at a density of $10^{-13} \mathrm{~g} / \mathrm{cm}^{3}$ is due to the chemical heating produced by the formation of $\mathrm{H}_{2}$ on grain surfaces. Dust cooling continues at high densities up to $10^{-10} \mathrm{~g} / \mathrm{cm}^{3}$ and after that the cloud becomes optically thick to dust self-absorption. Beyond this point the thermal evolution proceeds adiabatically and the inclusion of dust cooling breaks the a self-similarly of an isothermal collapse. A protostar is expected to form at this stage with a central adiabatic core and is expected to grow rapidly by accretion from the protostellar envelope.

For $\mathrm{Z} / \mathrm{Z}_{\odot}=10^{-5}$, the dust cooling becomes effective at densities of $10^{-14} \mathrm{~g} / \mathrm{cm}^{3}$, about two orders of magnitude higher than for $\mathrm{Z} / \mathrm{Z}_{\odot}=10^{-4}$ case. The temperature sharply declines down to a few hundred $\mathrm{K}$ and the $\mathrm{H}_{2}$ fraction gets boosted. The cloud becomes optically thick to dust cooling similar to the $\mathrm{Z} / \mathrm{Z}_{\odot}=10^{-4}$ case at $10^{-10} \mathrm{~g} / \mathrm{cm}^{3}$ and the thermal evolution follows the adiabatic equation of state. At an even lower metallicity of $\mathrm{Z} / \mathrm{Z}_{\odot}=10^{-6}$, the dust cooling becomes negligible and the thermal evolution follows the atomic cooling track. The $\mathrm{H}_{2}$ fraction is increased up to about a few times $10^{-4}$. The abundances of $\mathrm{e}^{-}$and $\mathrm{H}^{-}$remain very low and are similar at high densities for $\mathrm{Z} / \mathrm{Z}_{\odot}=10^{-5}$ and $\mathrm{Z} / \mathrm{Z}_{\odot}=10^{-4}$ cases. The degree of ionisation is higher for $\mathrm{Z} / \mathrm{Z}_{\odot}=10^{-6}$. The impact of metal line cooling such as CII and OI is negligible for such metallicities. Overall, our results are in good agreement with Omukai et al. (2008).

\subsection{D simulations}

In total, we have performed six cosmological simulations for two halos of $5.6 \times 10^{7} \mathrm{M}_{\odot}(\mathrm{H} 1)$ and $3.25 \times 10^{7} \mathrm{M}_{\odot}(\mathrm{H} 2)$ for metallicities of $\mathrm{Z} / \mathrm{Z}_{\odot}=$ $10^{-4}, 10^{-5}$ and $10^{-6}$. The collapse redshifts of the halos are 10.4 and 10.8, respectively, and independent of the dust content. We also performed one additional simulation with zero metallicity for the sake of comparison for H1. The gravitational collapse of the halos was followed from $1 \mathrm{Mpc}$ down to sub-AU scales with the adaptive mesh refinement approach. We assumed that the halos are irradiated by a strong LW flux of strength $10^{5}$ in terms of $\mathbf{J}_{21}$, well above the $\mathrm{J}_{\text {crit }}$ found in Latif et al. (2015), and the LW flux as well as the fixed metallicities of the above mentioned amount are turned on at $\mathrm{z}=30$.

\subsubsection{Thermodynamical and dynamical properties}

The density-temperature phase diagrams of both halos for $\mathrm{Z} / \mathrm{Z}_{\odot}=10^{-4}, 10^{-5}$ and $10^{-6}$ are shown in figure 2. In the presence of a strong LW flux which dissociates $\mathrm{H}_{2}$ molecules, the halos are unable to collapse via molecular hydrogen cooling and continue to grow via merging and accretion until they reach the atomic cooling limit. Both halos have recently gone through a major merger and the merger history of these halos is described in Latif \& Volonteri (2015). At densities of about $\geq 10^{-24} \mathrm{~g} / \mathrm{cm}^{3}$, atomic line cooling becomes effective and cools the gas to about $8000 \mathrm{~K}$. The collapse proceeds isothermally mainly via atomic hydrogen cooling up to densities of $10^{-16} \mathrm{~g} / \mathrm{cm}^{3}$ and the role of metal line cooling remains negligible. At densities $>10^{-16} \mathrm{~g} / \mathrm{cm}^{3}$, dust cooling comes into play and brings the gas temperature down to a few hundred $\mathrm{K}$ depending on the amount of dust. For $\mathrm{Z} / \mathrm{Z}_{\odot}=$ $10^{-6}$, the collapse remains isothermal up to densities of about $10^{-12} \mathrm{~g} / \mathrm{cm}^{3}$, at higher densities the dust cools the gas down to a temperature of $\sim 1000 \mathrm{~K}$ for halo 2 while both warm and cold phases coexist for halo 1 at densities $\geq 10^{-12} \mathrm{~g} / \mathrm{cm}^{3}$ due to the local variation in the gas density and collapse velocity. In the intermediate case of $\mathrm{Z} / \mathrm{Z}_{\odot}=10^{-5}$, the dust cooling becomes important earlier at densities of $\sim 10^{-14} \mathrm{~g} / \mathrm{cm}^{3}$ for both halos and decreases the gas temperature down to about $700 \mathrm{~K}$.

For $\mathrm{Z} / \mathrm{Z}_{\odot}=10^{-4}$, the dust grain cooling becomes strong at densities of $\sim 10^{-16} \mathrm{~g} / \mathrm{cm}^{3}$ and sharply brings the gas temperature down to a few hundred $\mathrm{K}$. The temperature stalls at about $1000 \mathrm{~K}$ at densities between $\sim 10^{-14}-10^{-12} \mathrm{~g} / \mathrm{cm}^{3}$ due to the chemical heating from the $\mathrm{H}_{2}$ formation on grain surfaces and again the dust cooling takes over at higher densities. These results are in agreement with the one-zone test presented in the previous section and also with Omukai et al. (2008). Small differences in the thermal evolution are observed for both halos, the cooling at high densities is generally more effective for halo 2 in comparison with halo 1 . We show the spherically averaged profiles of density, $\mathrm{H}_{2}$ fraction and temperature in figure 3 . The collapse remains isothermal down to the scales of $10,000 \mathrm{AU}$ irrespective of the metallicity explored in this work due to the strong LW flux. Below this scale dust cooling becomes important and remains confined to the central $10 \mathrm{AU}$ for $\mathrm{Z} / \mathrm{Z}_{\odot}=10^{-6}$, the inner $\sim 100$ $\mathrm{AU}$ for $\mathrm{Z} / \mathrm{Z}_{\odot}=10^{-5}$ and extends up to $3000 \mathrm{AU}$ for $\mathrm{Z} / \mathrm{Z}_{\odot}=10^{-4}$. The central temperature is about 300 , 700 and $1000 \mathrm{~K}$ for $\mathrm{Z} / \mathrm{Z}_{\odot}=10^{-4}-10^{-6}$, respectively. 
Similarly, the $\mathrm{H}_{2}$ fraction remains low at scales above $10^{5} \mathrm{AU}$ and exceeds $10^{-3}$ (above which $\mathrm{H}_{2}$ cooling becomes important) within the central part of the halo due to its formation on dust grains. For $\mathrm{Z} / \mathrm{Z}_{\odot}=10^{-4}$, the $\mathrm{H}_{2}$ abundance gets boosted within the central few $1000 \mathrm{AU}$ while for $\mathrm{Z} / \mathrm{Z}_{\odot}=10^{-5}$ only in the central few hundred $\mathrm{AU}$ and for $\mathrm{Z} / \mathrm{Z}_{\odot}=10^{-6}$ it remains confined to the central $10 \mathrm{AU}$.

The maximum density reached in our simulations is about $3 \times 10^{-11} \mathrm{~g} / \mathrm{cm}^{3}$ and the density increases with $\mathrm{R}^{-1.8}$ above $\sim 100 \mathrm{AU}$, close to the expected profile from an isothermal collapse (i.e. $\mathrm{R}^{-2.0}$ ). The density profile is shallower for $\mathrm{Z} / \mathrm{Z}_{\odot}=10^{-4}$ in the central $1000 \mathrm{AU}$ due to efficient dust cooling while for the other cases it almost follows the isothermal profile. Small bumps in the profile indicate the formation of additional clumps. The turbulent velocity is about $20 \mathrm{~km} / \mathrm{s}$ close to the viral radius and increases towards smaller scales up to about $30 \mathrm{~km} / \mathrm{s}$. This increase in the turbulent velocity is due to the infall of gas towards the center and is higher for dust cooling cases compared to the metal free case. To quantify whether a disk-like structure may form, we computed the ratio of the velocity dispersion (i.e., $\sqrt{c_{s}^{2}+v_{\text {turb }}^{2}}$ where $\mathrm{c}_{\mathrm{s}}$ is the sound speed and $\mathrm{v}_{\text {turb }}$ is the turbulent velocity) to the rotational velocity, corresponding to the vertical turbulent support within the disk. The latter provides an estimate for the ratio of vertical scale height to the disk radius. We found that this ratio remains about 1 for all cases except for halo 2 with $\mathrm{Z} / \mathrm{Z}_{\odot}=10^{-5}$ where $\mathrm{V}_{\text {rot }} / \sigma \sim 0.3$. This suggests that the rotational velocity is almost comparable to the velocity dispersion and a very thick disk-like structure with $H / R$ of about 1 is formed in all cases. To further estimate the rotational support against gravity, we calculated the ratio of rotational velocity to the Keplerian velocity $\left(\mathrm{V}_{\text {rot }} / \mathrm{V}_{\mathrm{Kep}}\right)$. The ratio of $\mathrm{V}_{\text {rot }} / \mathrm{V}_{\mathrm{Kep}}$ is shown in figure 4 and is about 0.6 for all cases. It gets enhanced between $60-1000$ $\mathrm{AU}$ for dust cooling cases. A peak in $\mathrm{V}_{\text {rot }} / \mathrm{V}_{\mathrm{Kep}}$ for $\mathrm{Z} / \mathrm{Z}_{\odot}=10^{-5}$ case comes from the density structure inside the halo. Overall, the estimates of $\mathrm{V}_{\text {rot }} / \mathrm{V}_{\mathrm{Kep}}$ suggest that the rotational support gets enhanced earlier for higher metallicity cases. However, enhanced rotational support can delay the runaway collapse but does not stop it. Moreover, the rotational support is only important in the envelope of the halo and decreases down within the central core.

\subsubsection{Mass inflow rates}

Our estimates for the mass inflow rates $\left(4 \pi R^{2} \rho v_{\text {rad }}\right)$ are shown in figure 3 Mass inflow rates of $0.1-$ $1 \mathrm{M}_{\odot} / \mathrm{yr}$ are observed for $\mathrm{Z} / \mathrm{Z}_{\odot} \leq 10^{-5}$ down to about $100 \mathrm{AU}$ while for $\mathrm{Z} / \mathrm{Z}_{\odot}=10^{-4}$ the mass inflow rate starts to decline around $10^{5} \mathrm{AU}$ for halo 2 . For comparison, we also show a zero-metallicity case where cooling is mainly due to atomic line cooling. We found that apart from small differences, the inflow rates for $\mathrm{Z} / \mathrm{Z}_{\odot} \leq 10^{-5}$ almost follow the zero-metallicity case. Below $100 \mathrm{AU}$ the mass inflow rates decline in all cases. It comes from the fact that during the runaway collapse a core-envelope structure develops, where the core has flat density and the envelope has $\mathrm{a} \sim \mathrm{R}^{-2}$ profile, with a core-envelope boundary around 10-100 $\mathrm{AU}$. The radial velocity is high inside the envelope, decreases down in the core and consequently a decline in the instantaneous inflow rate occurs towards the centre. In the central core thermal pressure balances the gravity as the core is not yet gravitationally unstable which results in a decline of the inflow rate. However, this may change at later stages when the central core further collapses. Fragmentation observed in the $\mathrm{Z} / \mathrm{Z}_{\odot}=10^{-4}$ cases may also partly contribute to the decline in the inflow rates. We also note that mass inflow rate scales with sound speed cube $\left(\dot{M} \propto c_{s}^{3}\right)$ and therefore decreases with the gas temperature. The dust cooling reduces the gas temperature which results in lower inflow rates. The accreting matter inside the envelope will collapse into the protostar at later times and high inflow rates are expected to be maintained. The increase in the mass inflow rates was observed in our previous simulations for a metal free case (Latif et al. 2013a) as more mass collapsed into the core. We expect a similar trend for other cases until the feedback from the central star blows away the gas or rotational support inhibits the mass accretion.

A similar trend is observed in the radial infall velocity which peaks around $15 \mathrm{~km} / \mathrm{s}$ and brings large inflows into the centre of the halo. The radial velocity temporarily becomes positive in a few places due to the presence of clumps, and the positive sign then describes the flow towards the other clump. This is particularly prominent in halo 2 for $\mathrm{Z} / \mathrm{Z}_{\odot}=10^{-4}$ and causes a decline in the mass inflow rate. Repeated peaks in the radial velocity indicate the onset of gravitational instabilities which help in the transfer of angular momentum. The enclosed gas mass in the central $30 \mathrm{pc}$ of the halo is a few times $10^{6} \mathrm{M}_{\odot}$. The mass profile increases with $\sim \mathrm{R}^{2}$ in the central $100 \mathrm{AU}$ and 
at larger scales linearly increases with radius. Some differences in the mass profile are observed between metal free and metal poor cases. The enclosed mass is a factor of a few lower for $\mathrm{Z} / \mathrm{Z}_{\odot}=10^{-4}$ in comparison with the metal-free case and is probably a consequence of differences in the thermal structure as well as enhanced rotation for $\mathrm{Z} / \mathrm{Z}_{\odot}=10^{-4}$ cases.

Overall, the mass inflow rates at the end of our simulations seem to be sufficient for forming a supermassive star at-least for $\mathrm{Z} / \mathrm{Z}_{\odot} \leq 10^{-5}$ while for higher metallicities enhanced rotation as well as fragmentation may limit accretion onto the central object. How long such accretion rates can be maintained depends on whether efficient fragmentation takes place or not. In the case of efficient fragmentation radiation feedback from in situ star formation may shut down accretion onto the central star and a supermassive star cannot form. Even in the absence of fragmentation, an enhanced rotation can limit mass accretion as found for $\mathrm{Z} / \mathrm{Z}_{\odot} \leq 10^{-4}$ in both halos. We discuss these possibilities in the section below.

\subsubsection{Fragmentation}

To quantify the fragmentation, we use the YT clump finder (Turk et al.2011) to identify clumps. Our clump finding algorithm locates topologically disconnected structures. The clumps are considered to be bound if the sum of kinetic and thermal energy is less than the potential energy, see Smith et al. (2009). The density structure within the central $1 \mathrm{pc}$ of the halo is shown in the figure 5 for all cases. There is not much substructure found at this scale as the collapse is almost isothermal and dust cooling starts to become important around a few $1000 \mathrm{AU}$. Zooming into the central $4000 \mathrm{AU}$ (see figure 6), significant differences in the morphology of the halos are observed. Particularly, for $\mathrm{Z} / \mathrm{Z}_{\odot}=10^{-4}$, the structure is more elongated and collapses into a filament due to the more efficient cooling compared with other cases where central gas clouds are more spherical. For $\mathrm{Z} / \mathrm{Z}_{\odot}=10^{-4}$, two well separated gravitationally bound clumps of about a solar mass are formed in halo 2 while in halo 1 the central clump is of similar mass, gravitationally bound but the second clump is gravitationally unbound and has a sub-solar mass. In contrast to this, the clouds are more spherical (although not completely) for $\mathrm{Z} / \mathrm{Z}_{\odot}=10^{-6}$, very similar to a zero metal case.

For $\mathrm{Z} / \mathrm{Z}_{\odot}=10^{-5}$, there is more substructure in the central part of halo 1 with the possibility of multi- ple clump formation while halo 2 looks similar to the metal free case. Overall the clouds are more elongated compared to the $\mathrm{Z} / \mathrm{Z}_{\odot}=10^{-6}$. We also ran a clump finder for these cases and found that all clumps in these cases are gravitationally unbound and mostly with subsolar masses as shown in figure 8. The figure 7 shows that only small pockets of gas are cooled by dust cooling. The $\mathrm{Z} / \mathrm{Z}_{\odot}=10^{-6}$ case looks very similar to the metal free case, the temperature of the dense clumps of gas is about a thousand $\mathrm{K}$ for $\mathrm{Z} / \mathrm{Z}_{\odot}=10^{-5}$ and it declines down to a few hundred $\mathrm{K}$ for $\mathrm{Z} / \mathrm{Z}_{\odot}=10^{-4}$. Similar differences in the density structure of a gas cloud are observed by Peters et al. (2012) employing an equation of state with polytropic index $(\gamma<) 1$.

We estimated the maximum scale of thermal instability (i.e. $l_{\text {th }}=c_{\mathrm{s}} \mathrm{t}_{\text {cool }}$ ) following the criterion given in Inoue \& Omukai (2015) and found that it is comparable to the Jeans length. It shows that the resolution in our simulations is enough to resolve the thermal instability. Dust cooling triggers the Jeans instability which results in the formation of multiple clumps. In a self-gravitating cloud, turbulence can also induce fragmentation by locally compressing the gas as observed in an isothermal collapse, see Latif et al. (2013a). Overall, central clumps forming for $\mathrm{Z} / \mathrm{Z}_{\odot}=10^{-4}$ are gravitationally bound and for low metallicities all the clumps are gravitationally unbound. We caution the reader that gravitationally unbound clumps at the initial stages of the collapse do not reflect the true amount of fragmentation. To further estimate the fate of these clumps, we followed the collapse of halo 2 with $\mathrm{Z} / \mathrm{Z}_{\odot}=10^{-5}$ to densities of $10^{-8} \mathrm{~g} / \mathrm{cm}^{3}$ by employing 5 additional refinement levels. In this case, clumps eventually collapse into the centre of the halo. However, the possibility of fragmentation at later stages of the collapse cannot be ruled out and it may vary from halo to halo. Evolving these simulations further in time becomes computationally extremely expensive due to the large dynamical range covered in these simulations which results in very short time steps. In the following section, we discuss the possible implications of efficient fragmentation if it occurs at later times.

\subsection{Fraction of metal enriched halos}

We estimate the fraction of metal polluted halos with $\mathrm{Z} / \mathrm{Z}_{\odot} \leq 10^{-4}$ from cosmological hydrodynamical simulations Habouzit et al. (2016). The computational volume has a size of $10 \mathrm{Mpc}$ and simulations include recipes for star formation and super- 
nova feedback, a detailed discussion is provided in Habouzit et al. (2016). We identified the halos with mass range between $2 \times 10^{7}-10^{8} \mathrm{M}_{\odot}$ using the HaloMaker code (Tweed et al. 2009) at least resolved by 100 hundred DM particles and computed their mean metallicity. Our estimates for the fraction of halos with $\mathrm{Z} / \mathrm{Z}_{\odot} \leq 10^{-4}$ are shown in figure 9 . The number of metal polluted halos increases with decreasing redshift as expected and the fraction of halos polluted with $\mathrm{Z} / \mathrm{Z}_{\odot} \leq 10^{-5}$ is about a factor of 1.5 higher in comparison with metal free halos.

The halos polluted by a trace amount of metals can be irradiated by a strong LW flux if they form in the close vicinity of a star forming galaxy. Our simulations do not self-consistently take into account the local variations in the LW flux required for the direct collapse sites and the simulations volume is not large enough to capture rare sources which provide a strong LW flux of $10^{5}$ in terms of $J_{21}$. Assuming that the metallicity distribution is however uncorrelated with fluctuations in the LW background, if a direct collapse is feasible for metallicities up to $10^{-5}$, the resulting abundance of the halos is increased by a factor of 1.5 compared to the purely primordial case.

\section{Implications for black holes formation}

Stellar evolution calculations suggest that the main requirement for the formation of direct collapse black holes are mass inflow rates of $\geq 0.1 \mathrm{M}_{\odot} / \mathrm{yr}$. For such accretion rates, stellar evolution differs from normal stars, as the stellar radius increases with mass and no strong UV feedback is produced by the protostar. Under these conditions, a supermassive star of about $10^{5} \mathrm{M}_{\odot}$ can be formed which may later collapse into a black hole while retaining most of its mass (Hosokawa et al. 2012, 2013; Schleicher et al. 2013).

In this study, we explored the impact of dust cooling occurring at high densities in the presence of a strong LW flux for two halos of a few times $10^{7} \mathrm{M}_{\odot}$ and metallicities of $\mathrm{Z} / \mathrm{Z}_{\odot}=10^{-4}-10^{-6}$. We resolved the collapse down to sub-AU scales and densities of about a few times $10^{-11} \mathrm{~g} / \mathrm{cm}^{3}$. The presence of large mass inflow rates of $\geq 0.1 \mathrm{M}_{\odot} / \mathrm{yr}$ suggests that trace amounts of metals and dust do not inhibit the formation of massive objects. At least for $\mathrm{Z} / \mathrm{Z}_{\odot} \leq 10^{-5}$, the mass accretion rates are comparable with an atomic cooling case and no strong fragmentation is observed. It should be noted that the conditions found here are different from minihalos cooled by dust cooling in the absence of a strong LW background. Both mass inflow rates and available gas mass are about two orders of magnitude higher compared to minihalos. Due to the extremely large spatial range covered in our simulations, the time step becomes very short and does not allow us to evolve simulations long enough to assess fragmentation and inflow rates at later times. In the following, we consider two possible outcomes assuming that efficient fragmentation occurs at later times.

In the first case, we consider that efficient fragmentation occurs at later times but clumps move inward due to the short migration time scale and merge with the central clump (Inayoshi \& Haiman 2014; Latif \& Schleicher 2015a b; Schleicher et al. 2015). In the presence of the large inflow rates of $0.1 \mathrm{M}_{\odot} / \mathrm{yr}$ found here, viscous heating may become important in the interior of the disk, it will heat the gas up to a few thousand K. Therefore, viscous heating may suppress fragmentation by increasing the thermal Jeans mass and may help in the formation of massive objects (Latif \& Schleicher 2015b; Schleicher et al. 2015). Moreover, for such large inflow rates the impact of UV feedback from stars is expected to be quite weak. Even if some of the clumps survive or get ejected via 3-body processes and form low mass stars, a massive central object is still expected to form due to the clumpy mode of accretion and the stabilisation of the disk due to the viscous heating. We argue that in such a scenario, massive seed black holes of $\sim 10^{4} \mathrm{M}_{\odot}$ can form for $\mathrm{Z} / \mathrm{Z}_{\odot} \leq 10^{-5}$.

For the second case, we assume that efficient fragmentation takes place due to the dust cooling at high densities. Then the clumps are unable to migrate inward and a stellar cluster forms. A dense stellar cluster is expected to form in such a scenario as dust cooling becomes effective only in the central few 1001000 AU surrounded by hot gas with a temperature around $8000 \mathrm{~K}$. The minimum Jeans mass at high densities where dust cooling operates in our simulations is about $>0.1 \mathrm{M}_{\odot}$ and the enclosed mass within a radius of a few hundred $\mathrm{AU}$ is about $1000 \mathrm{M}_{\odot}$. As a consequence of gravitational collapse, a higher gas mass will collapse in the centre over time and may reach up to $10^{6} \mathrm{M}_{\odot}$ which is about few $\%$ of the halo mass as often observed in numerical simulations (Johnson et al. 2009; Latif et al. 2011). For a star formation efficiency of about $10 \%$, a stellar cluster of about $10^{5} \mathrm{M}_{\odot}$ may form. The expected radius of such a stellar cluster is about 1000 AU. Various studies suggest that such dense clusters may collapse into a 
massive black hole either via relativistic instabilities or stellar dynamical processes (Baumgarte \& Shapiro 1999; Portegies Zwart et al. 1999; Omukai et al. 2008; Devecchi \& Volonteri 2009; Devecchi et al.2012). We estimate the mass of a seed black hole forming from the core collapse of a cluster in the following way (Portegies Zwart \& McMillan 2002),

$$
M_{B H}=m_{*}+4 \times 10^{-3} f_{c} M_{c 0} \gamma \ln \Lambda_{C},
$$

where $\mathrm{m}_{*}$ is the mass of a massive star in the cluster which initiates runaway growth, $f_{c}$ is the effective fraction of binaries formed dynamically, $\mathrm{M}_{\mathrm{c} 0}$ is the mass of the cluster at its birth, $\ln \Lambda_{c}$ is the Coulomb logarithm and $\gamma \sim 1$, is the ratio of various time scales, see Portegies Zwart \& McMillan (2002). For a star cluster of $\mathrm{M}_{\mathrm{c} 0}=10^{4} \mathrm{M}_{\odot}, \mathrm{m}_{*}=100 \mathrm{M}_{\odot}, \mathrm{f}_{\mathrm{c}}=0.2, \ln \Lambda_{C}=10$, we get $\mathrm{M}_{\mathrm{BH}} \sim 180 \mathrm{M}_{\odot}$. For a $\mathrm{M}_{\mathrm{c} 0}=10^{5} \mathrm{M}_{\odot}$, expected black holes mass is $\sim 900 \mathrm{M}_{\odot}$. Therefore, depending on the initial mass of a cluster and the central star, massive black holes seeds of up to a thousand solar masses can be formed in metal-poor halos illuminated by the strong LW flux.

\section{Discussion and conclusions}

We have performed high resolution cosmological simulations to study the impact of trace amounts of metals and dust cooling in massive primordial halos irradiated by the strong LW flux. To accomplish this goal, we selected two massive halos of a few times $10^{7} \mathrm{M}_{\odot}$ at $z>10$ with metallicities of $\mathrm{Z} / \mathrm{Z}_{\odot}=10^{-4}$ $10^{-6}$ and turned on a strong LW radiation of strength $10^{5}$ in terms of $\mathrm{J}_{21}$. Our simulations cover a large dynamical range by resolving the collapse starting from cosmological scales down to scales of sub-AU. To take into account the effect of dust and metal line cooling, we extended our previous chemical model and included $\mathrm{H}_{2}$ formation on dust grains, dust grain cooling and heating, CII and OI metal lines cooling following Omukai et al. (2008).

Our results show that even in the presence of a trace amount of metals and dust, the collapse proceeds isothermally with temperatures around $8000 \mathrm{~K}$ up to densities of about $10^{-16} \mathrm{~g} / \mathrm{cm}^{3}$. Dust cooling becomes effective at densities between $10^{-16}-10^{-12} \mathrm{~g} / \mathrm{cm}^{3}$ and brings the gas temperature down to $100-1000 \mathrm{~K}$ for $\mathrm{Z} / \mathrm{Z}_{\odot}=10^{-4}-10^{-6}$, respectively. As expected, dust cooling is more efficient and occurs at earlier stages of the collapse for higher metallicities in comparison with a lower metallicity case. In contrast to the isothermal case, $\mathrm{H}_{2}$ formation on dust grains takes place in the core of the halo and its fraction gets boosted. For the metal poor cases studied here, metal line cooling is not important but could be effective at metallicities higher than explored here.

We found that large inflow rates of $0.1 \mathrm{M}_{\odot} / \mathrm{yr}$ are available for $\mathrm{Z} / \mathrm{Z}_{\odot} \leq 10^{-5}$ while for the higher metallicity case the inflow rates start to decline earlier. The decline in accretion rate for higher metallicity cases is due to the lower gas temperature in the presence of dust cooling as well as because of the fragmentation. No gravitationally bound clumps are found for $\mathrm{Z} / \mathrm{Z}_{\odot} \leq 10^{-5}$ by the end of our simulations while for $\mathrm{Z} / \mathrm{Z}_{\odot}=10^{-4}$ clumps are gravitationally bound and more massive. The morphology of the central gas cloud is significantly different in $\mathrm{Z} / \mathrm{Z}_{\odot}=10^{-4}$, as the cloud is more elongated compared to other cases probably due to the more efficient dust cooling. Larson (1969) and Penston (1969) found a self-similar solution under isothermal conditions and later Yahil (1983) showed that it changes with the equation of state by including a dependence of the collapse time on the equation of state parameter $\gamma=1+\frac{\operatorname{dog} \rho}{\operatorname{dlog} T}$. The latter suggests that the collapse dynamics will be changed by adding dust cooling. Overall, no strong fragmentation is observed for $\mathrm{Z} / \mathrm{Z}_{\odot} \leq 10^{-5}$ cases although the possibility of fragmentation at later stages of the collapse cannot be ruled out. Due to the computational constraints, we were unable to evolve simulations for longer times and therefore cannot make strong statements about fragmentation occurring at later times.

The presence of large inflow rates suggests that a massive central star is expected to form for $\mathrm{Z} / \mathrm{Z}_{\odot} \leq$ $10^{-5}$ where dust cooling is confined to the central 10-100 AU depending on the metallicity. Even if the cloud fragments at later stages of the collapse, the clumps are expected to migrate inward and merge in the centre as proposed by theoretical works (Inayoshi \& Haiman 2014; Latif \& Schleicher 2015b). Viscous heating which becomes particularly important in the presence of large inflow rates and rapid rotation may further help in suppressing the fragmentation by heating the gas and evaporating the dust grains (Latif \& Schleicher 2015b; Schleicher et al. 2015). For $\mathrm{Z} / \mathrm{Z}_{\odot}=10^{-4}$, efficient fragmentation is expected to occur which may lead to the formation of a stellar cluster.

The strength of the LW flux required to keep the collapse completely isothermal in metal-free halos is about a few times $10^{4}$ (Latif et al. 2015) and in the 
present work we employed even a higher value of $10^{5}$ in units of $\mathbf{J}_{21}$. The expected number density of direct collapse black holes forming in primordial halos for $\mathrm{J}_{\text {crit }} \geq 10^{4}$ is a few orders of magnitude lower than the observed abundance of quasars at $\mathrm{z}=6$ (i.e. 1 per $\mathrm{Gpc}^{3}$ (Willott et al. 2010)) extrapolating the results of Habouzit et al. (2015). Even relaxing the constraint of primordial gas and allowing the direct collapse black holes to form in halos with $\mathrm{Z} / \mathrm{Z}_{\odot} \leq 10^{-5}$ in the presence of a strong LW flux only increases their abundance by a factor of two. Even under these conditions, the formation sites for the direct collapse black holes remain rare.

For metallicities higher than explored here, cooling due to the fine structure lines of CII and OI becomes important at low densities about $10^{-20} \mathrm{~g} / \mathrm{cm}^{3}$ even in the presence of a strong LW flux and cools the gas down to a few $10 \mathrm{~K}$. In such cases, the thermal instability induces fragmentation and determines the mass spectrum of the resulting star cluster (Inoue \& Omukai 2015).

\section{Acknowledgments}

This project has received funding from the European Union's Horizon 2020 research and innovation programme under the Marie Sklodowska-Curie grant agreement $\mathrm{N}^{o}$ 656428. The research leading to these results has also received funding from the European Research Council under the European Community's Seventh Framework Programme (FP7/2007-2013 Grant Agreement no. 614199, project "BLACK"). KO acknowledges the Grant-in-aids from the Ministry of Education, Culture, Sports, Science, and Technology (MEXT) of Japan (25287040). This work was granted access to the HPC resources of TGCC under the allocation x2015046955 made by GENCI. The simulation results are analyzed using the visualization toolkit for astrophysical data YT (Turk et al. 2011).

\section{REFERENCES}

Abel, T., Bryan, G. L., \& Norman, M. L. 2002, Science, 295, 93

Agarwal, B., \& Khochfar, S. 2015, MNRAS, 446, 160

Agarwal, B., Khochfar, S., Johnson, J. L., Neistein, E., Dalla Vecchia, C., \& Livio, M. 2012, MNRAS, 425, 2854
Baumgarte, T. W., \& Shapiro, S. L. 1999, ApJ, 526, 941

Becerra, F., Greif, T. H., Springel, V., \& Hernquist, L. E. 2015, MNRAS, 446, 2380

Begelman, M. C. 2010, MNRAS, 402, 673

Begelman, M. C., \& Shlosman, I. 2009, ApJ, 702, L5

Begelman, M. C., Volonteri, M., \& Rees, M. J. 2006, MNRAS, 370, 289

Bovino, S., Grassi, T., Schleicher, D. R. G., \& Latif, M. A. 2014, ApJ, 790, L35

Bromm, V., \& Loeb, A. 2003, ApJ, 596, 34

Bryan, G. L., et al. 2014, ApJS, 211, 19

Cazaux, S., \& Spaans, M. 2009, A\&A, 496, 365

Choi, J.-H., Shlosman, I., \& Begelman, M. C. 2015, MNRAS, 450, 4411

Davies, M. B., Miller, M. C., \& Bellovary, J. M. 2011, ApJ, 740, L42

Devecchi, B., \& Volonteri, M. 2009, ApJ, 694, 302

Devecchi, B., Volonteri, M., Rossi, E. M., Colpi, M., \& Portegies Zwart, S. 2012, MNRAS, 421, 1465

Dijkstra, M., Ferrara, A., \& Mesinger, A. 2014, MNRAS, 442, 2036

Dijkstra, M., Haiman, Z., Mesinger, A., \& Wyithe, J. S. B. 2008, MNRAS, 391, 1961

Dopcke, G., Glover, S. C. O., Clark, P. C., \& Klessen, R. S. 2013, ApJ, 766, 103

Fan, X., Strauss, M. A., Richards, G. T., Hennawi, J. F., Becker, R. H., White, R. L., \& DiamondStanic, A. M. 2006, AJ, 131, 1203

Federrath, C., Sur, S., Schleicher, D. R. G., Banerjee, R., \& Klessen, R. S. 2011, ApJ, 731, 62

Ferrara, A., Salvadori, S., Yue, B., \& Schleicher, D. 2014, MNRAS, 443, 2410

Glover, S. C. O., \& Jappsen, A.-K. 2007, ApJ, 666, 1

Grassi, T., Bovino, S., Schleicher, D. R. G., Prieto, J., Seifried, D., Simoncini, E., \& Gianturco, F. A. 2014, MNRAS, 439, 2386 

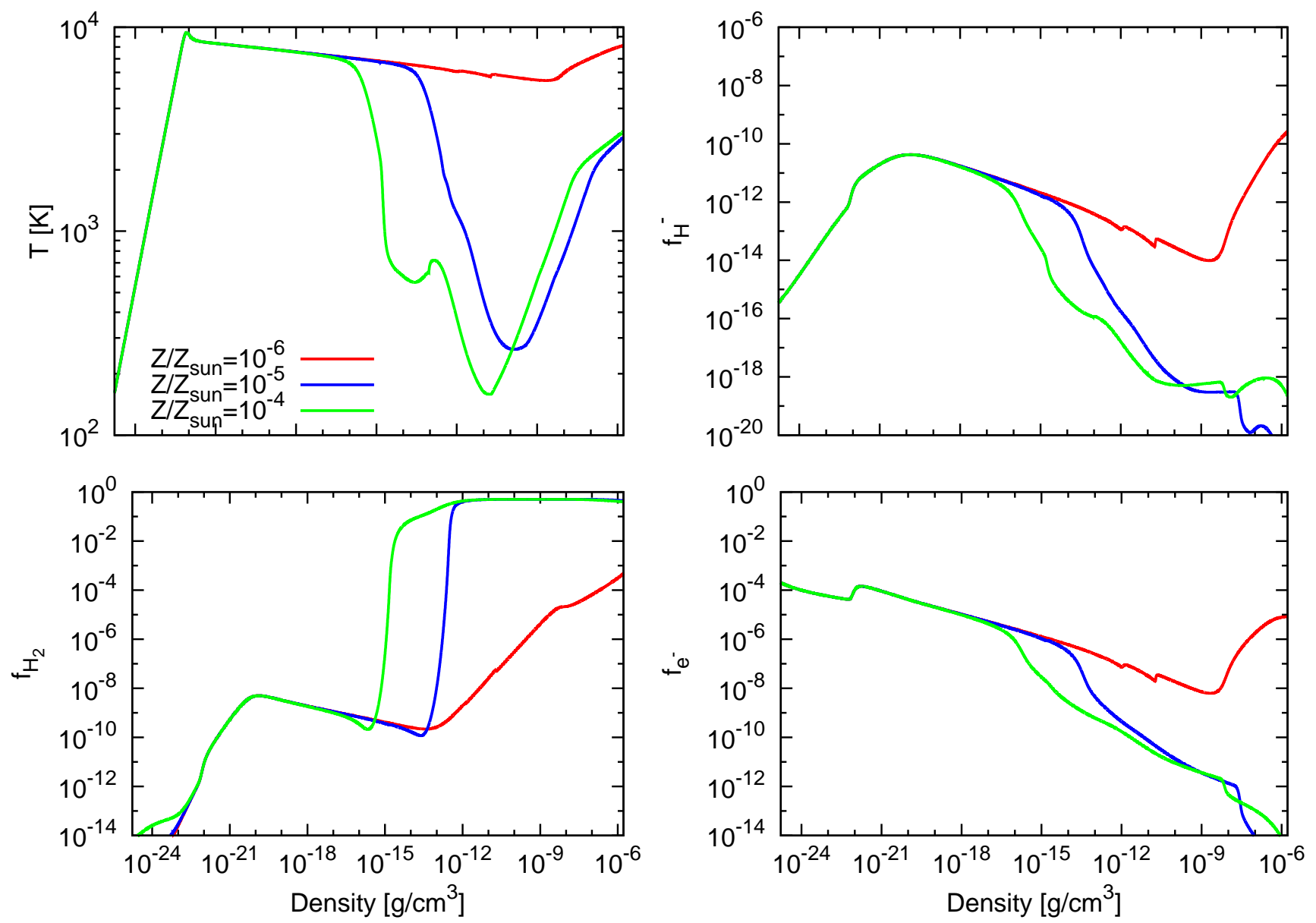

Fig. 1.- Figure shows the temperature, $\mathrm{H}_{2}, \mathrm{H}^{-}$and $\mathrm{e}^{-}$fractions plotted against the density from one-zone test. The green, blue and red lines represent $\mathrm{Z} / \mathrm{Z}_{\odot}=10^{-6}, 10^{-5}$ and $10^{-4}$, respectively. 


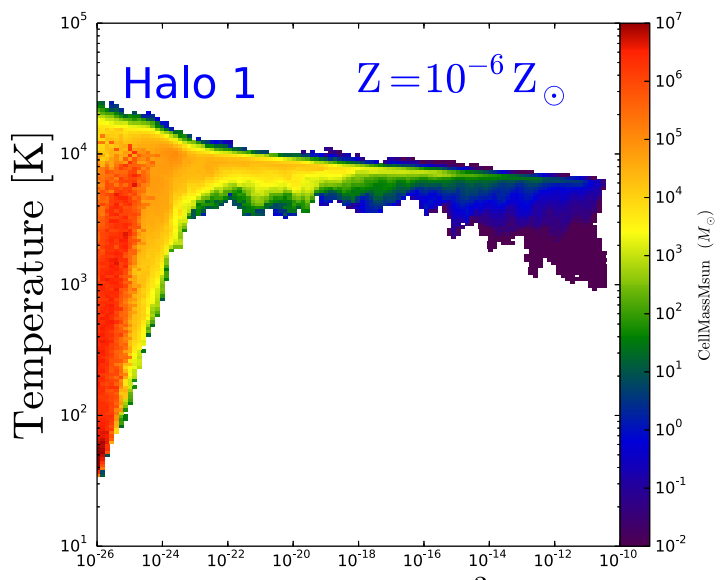

Density $\left[\mathrm{g} / \mathrm{cm}^{3}\right]$

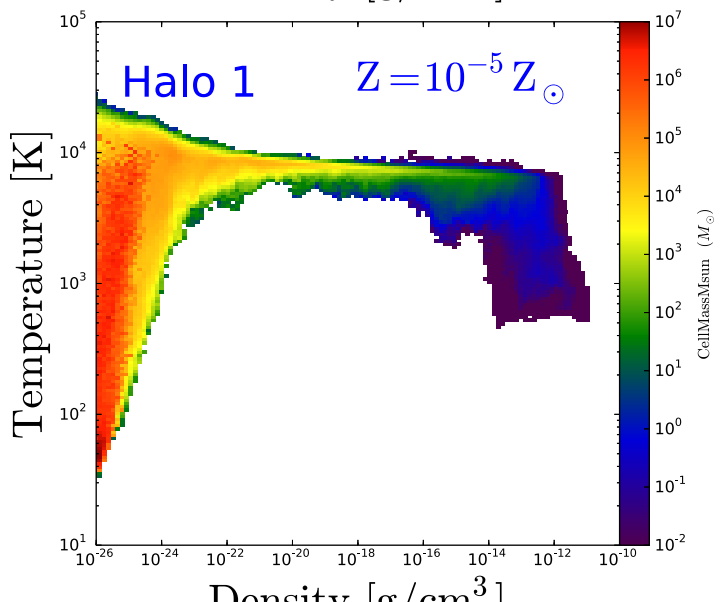

Density $\left[\mathrm{g} / \mathrm{cm}^{3}\right]$

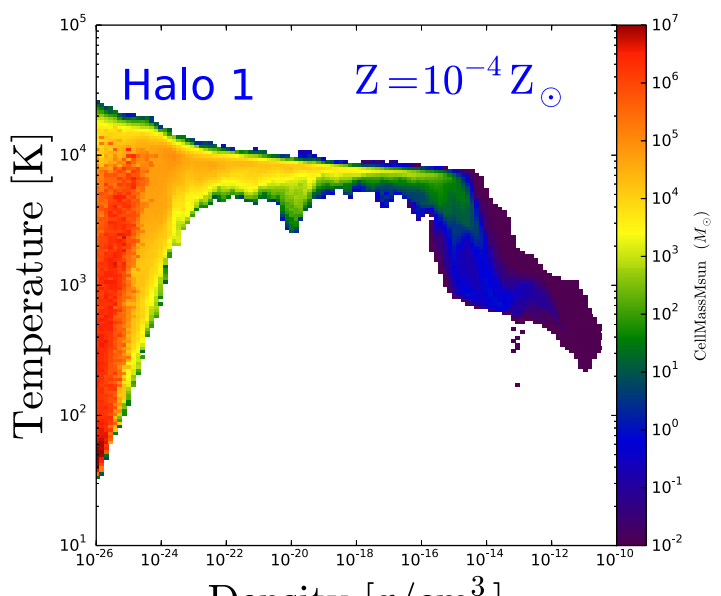

Density $\left[\mathrm{g} / \mathrm{cm}^{3}\right.$ ]

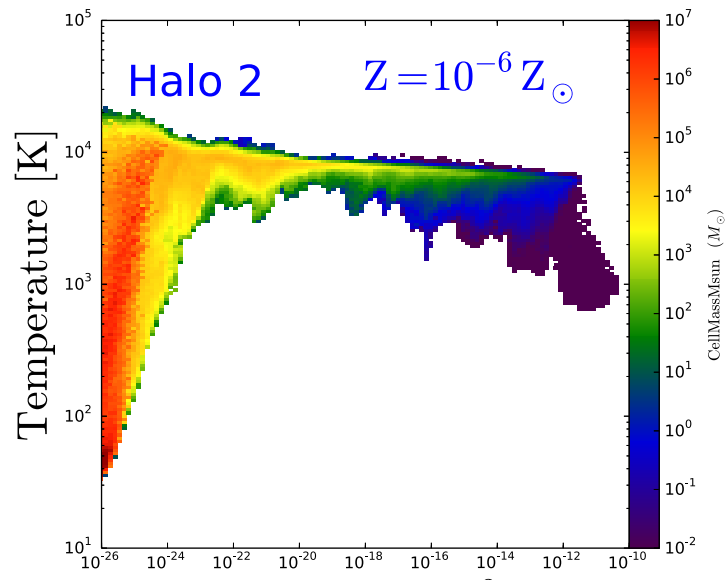

Density $\left[\mathrm{g} / \mathrm{cm}^{3}\right]$

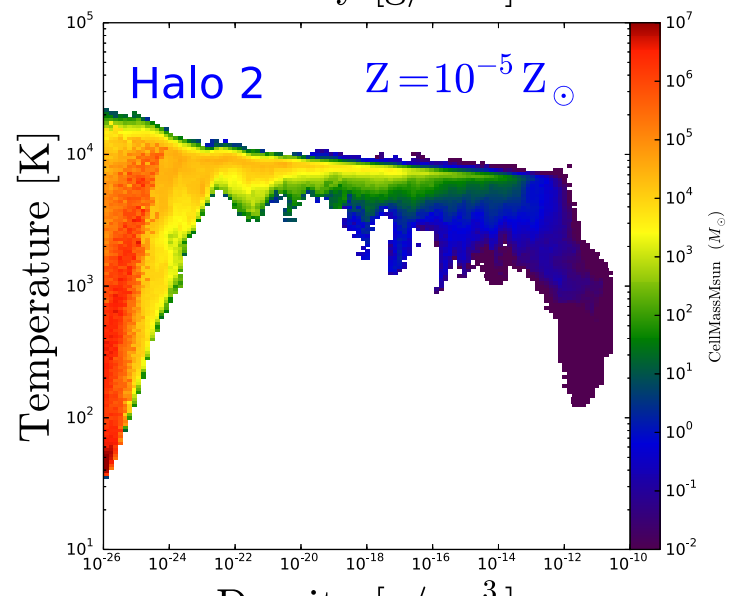

Density $\left[\mathrm{g} / \mathrm{cm}^{3}\right\rceil$

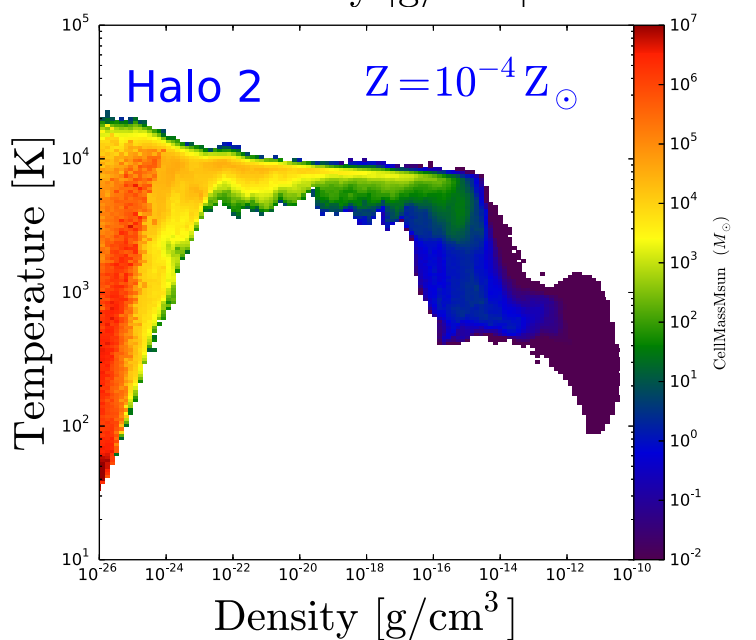

Fig. 2.- Density-temperature phase diagram for $\mathrm{Z} / \mathrm{Z}_{\odot}=10^{-6}, 10^{-5}$ and $10^{-4}$ from top to bottom, respectively. The left panel represents halo 1 and the right panel halo 2 . The gas is initially heated up to a few times $10^{4} \mathrm{~K}$, collapses isothermally up to the densities of $10^{-16} \mathrm{~g} / \mathrm{cm}^{3}$ in the presence of a strong LW flux and the dust cooling becomes effective at $\geq 10^{-16} \mathrm{~g} / \mathrm{cm}^{3}$ densities depending on the amount of metallicity. Colour-bars show the amount of gas in solar masses for a given density. 

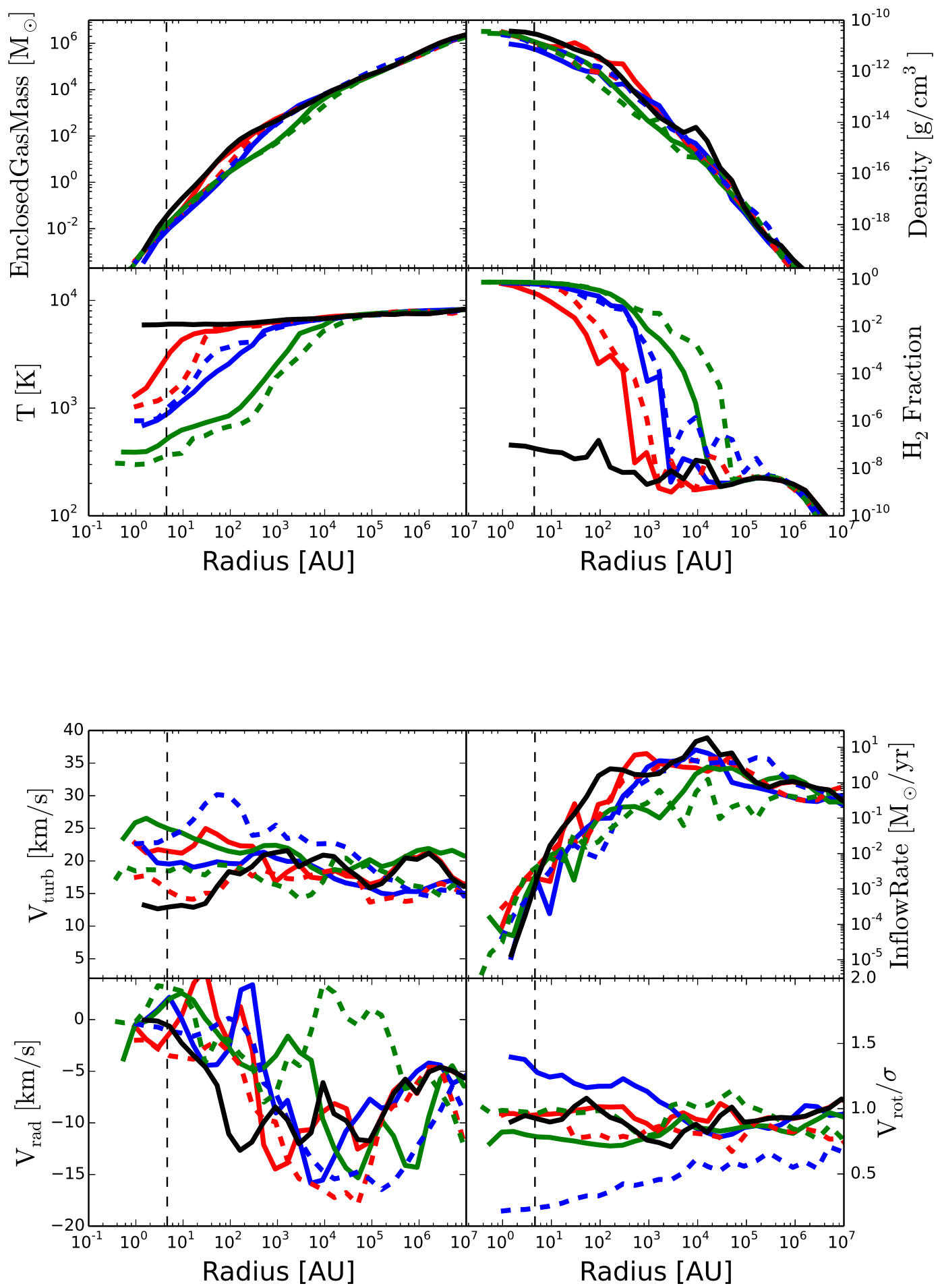

Fig. 3.- Radially averaged and spherically binned profiles of various quantities for halo 1 (H1) and halo 2 (H2) are shown here. The green, blue and red lines represent $\mathrm{Z} / \mathrm{Z}_{\odot}=10^{-4}, 10^{-5}$ and $10^{-6}$, respectively. The solid lines represent halo 1 and the dashed lines show halo 2. The vertical black dashed line corresponds to the Jeans length. An isothermal case for halo 1 with zero metallicity is also plotted as a reference and is represented by the black solid line. 


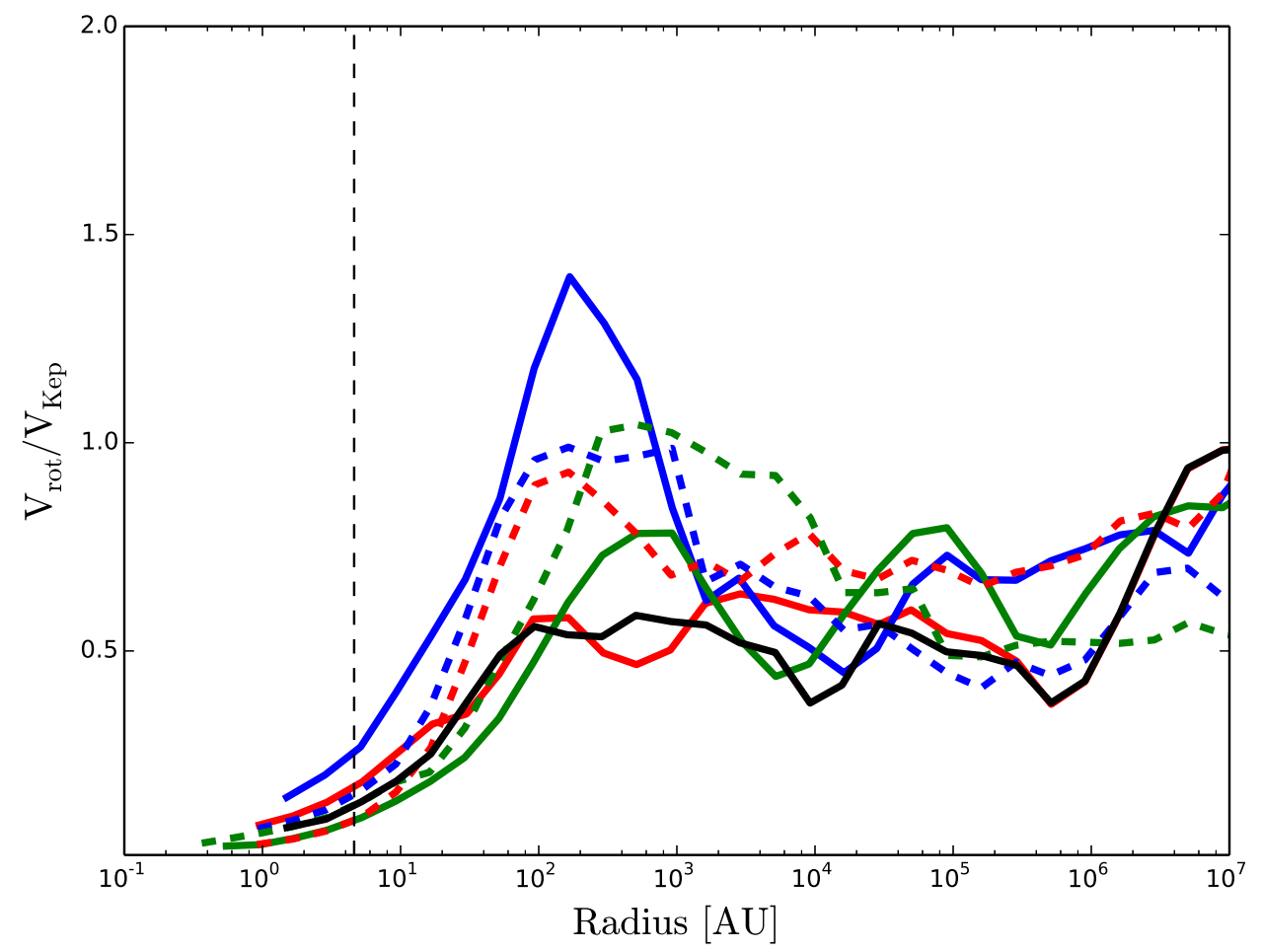

Fig. 4.- The ratio of rotational to Keplerian velocity for halo 1 (H1) and halo 2 (H2) is shown here. The green, blue and red lines represent $Z / Z_{\odot}=10^{-4}, 10^{-5}$ and $10^{-6}$, respectively. The solid lines represent halo 1 and the dashed lines show halo 2. An isothermal case for halo 1 with zero metallicity is also plotted as a reference and is represented by the black solid line. The vertical black dashed line corresponds to the Jeans length. 


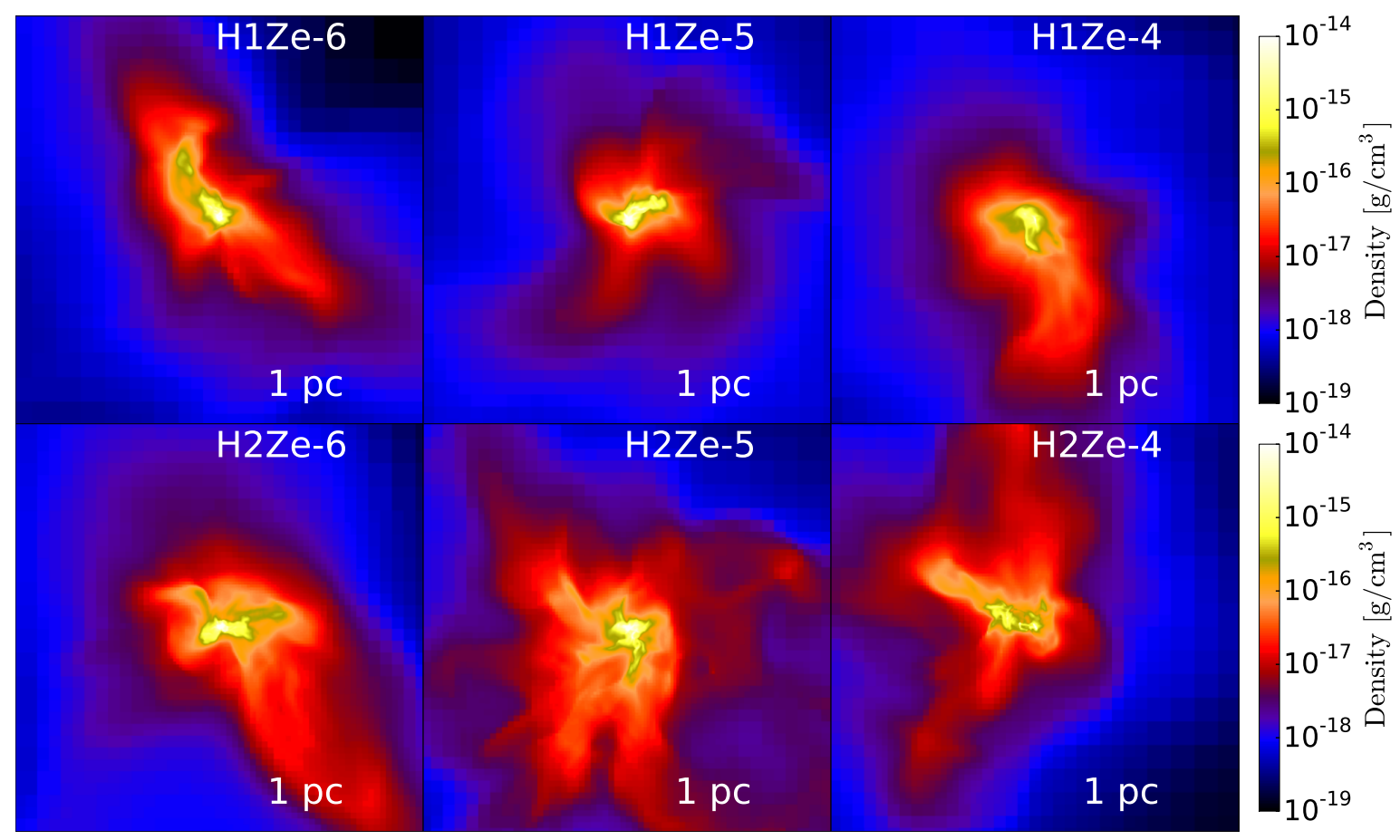

Fig. 5.- Average gas density along $\mathrm{x}$-axis for the central $1 \mathrm{pc}$ of a halo. Each row represents a halo (halo 1(H1) on top and halo 2 (H2) on bottom) and each column represent metallicity (increasing from left to right). H1Ze-6, H1Ze-5 and H1Ze-4 represent $Z / Z_{\odot}=10^{-6}, 10^{-5}$ and $10^{-4}$ for halo 1 , respectively. Similarly, H2Ze-6, H2Ze-5 and H2Ze-4 represent $\mathrm{Z} / \mathrm{Z}_{\odot}=10^{-6}, 10^{-5}$ and $10^{-4}$ for halo 2 . 


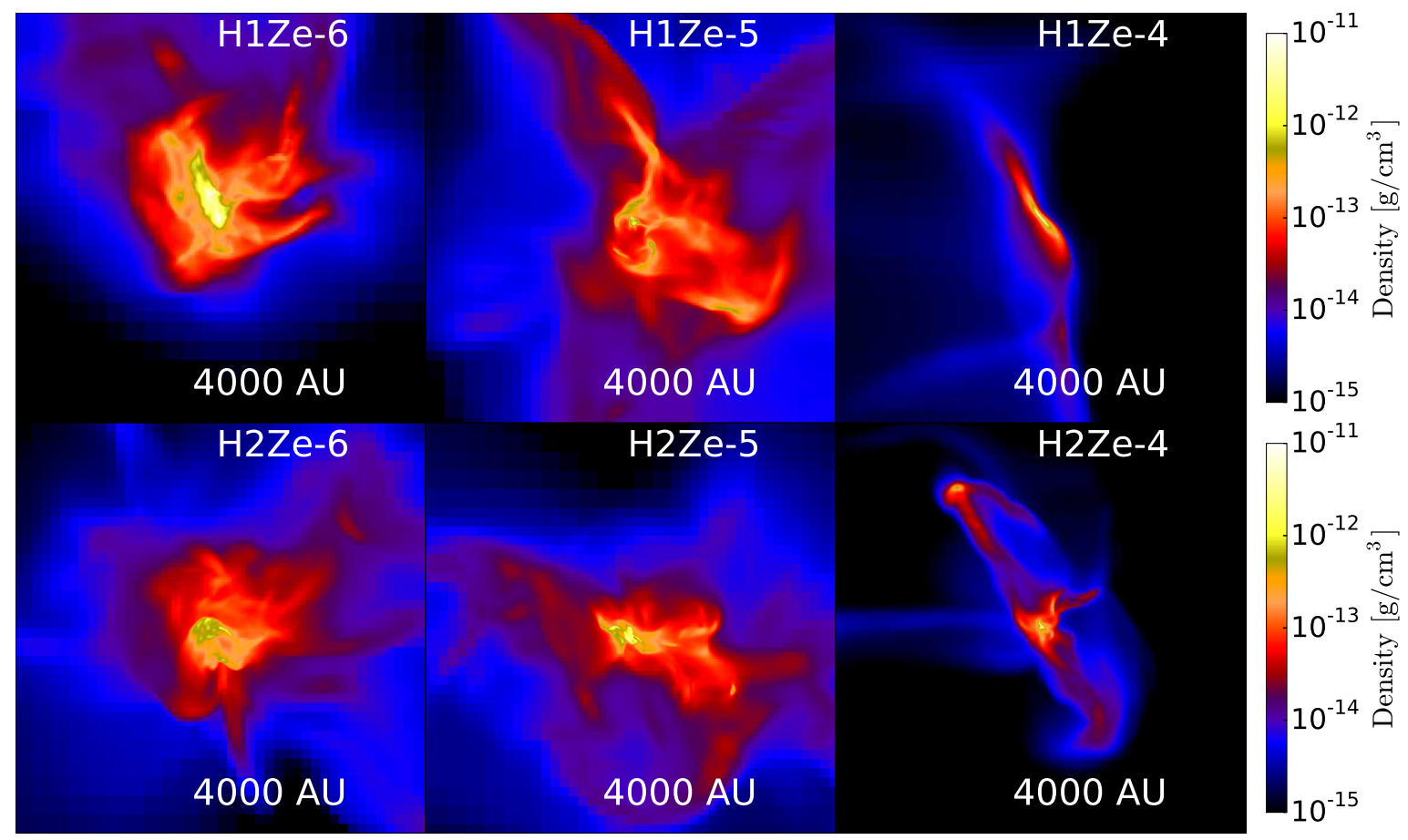

Fig. 6.- Average gas density along x-axis for the central $4000 \mathrm{AU}$ of a halo. Each row represents a halo (halo 1 on top and halo 2 on bottom) and each column represent metallicity (increasing from left to right).

Habouzit, M., Volonteri, M., Latif, M., Dubois, Y., \& Peirani, S. 2016, ArXiv e-prints:1601.00557

Habouzit, M., et al. 2015, ArXiv e-prints:1507.05971

Haiman, Z. 2012, ArXiv e-prints-1203.6075

Hirano, S., Hosokawa, T., Yoshida, N., Umeda, H., Omukai, K., Chiaki, G., \& Yorke, H. W. 2014, ApJ, 781,60

Hollenbach, D., \& McKee, C. F. 1979, ApJS, 41, 555

Hosokawa, T., Omukai, K., \& Yorke, H. W. 2012, ApJ, 756,93

Hosokawa, T., Yorke, H. W., Inayoshi, K., Omukai, K., \& Yoshida, N. 2013, ApJ, 778, 178

Inayoshi, K., \& Haiman, Z. 2014, MNRAS, 445, 1549

Inayoshi, K., Omukai, K., \& Tasker, E. 2014, MNRAS, 445, L109

Inoue, T., \& Omukai, K. 2015, ApJ, 805, 73

Jarosik, N., Bennett, C. L., Dunkley, J., Gold, B., Greason, M. R., Halpern, M., Hill, R. S., \& Hinshaw, G. 2011, ApJS, 192, 14
Johnson, J. L., Dalla Vecchia, C., \& Khochfar, S. 2013, MNRAS, 428, 1857

Johnson, J. L., Greif, T. H., Bromm, V., Klessen, R. S., \& Ippolito, J. 2009, MNRAS, 399, 37

Katz, H., Sijacki, D., \& Haehnelt, M. G. 2015, MNRAS, 451, 2352

Larson, R. B. 1969, MNRAS, 145, 271

Latif, M. A., Bovino, S., Grassi, T., Schleicher, D. R. G., \& Spaans, M. 2015, MNRAS, 446, 3163

Latif, M. A., Bovino, S., Van Borm, C., Grassi, T., Schleicher, D. R. G., \& Spaans, M. 2014, MNRAS, 443, 1979

Latif, M. A., \& Schleicher, D. R. G. 2015a, MNRAS, 449,77

-. 2015b, A\&A, 578, A118

Latif, M. A., Schleicher, D. R. G., \& Hartwig, T. 2016, MNRAS

Latif, M. A., Schleicher, D. R. G., Schmidt, W., \& Niemeyer, J. 2013a, MNRAS, 433, 1607 


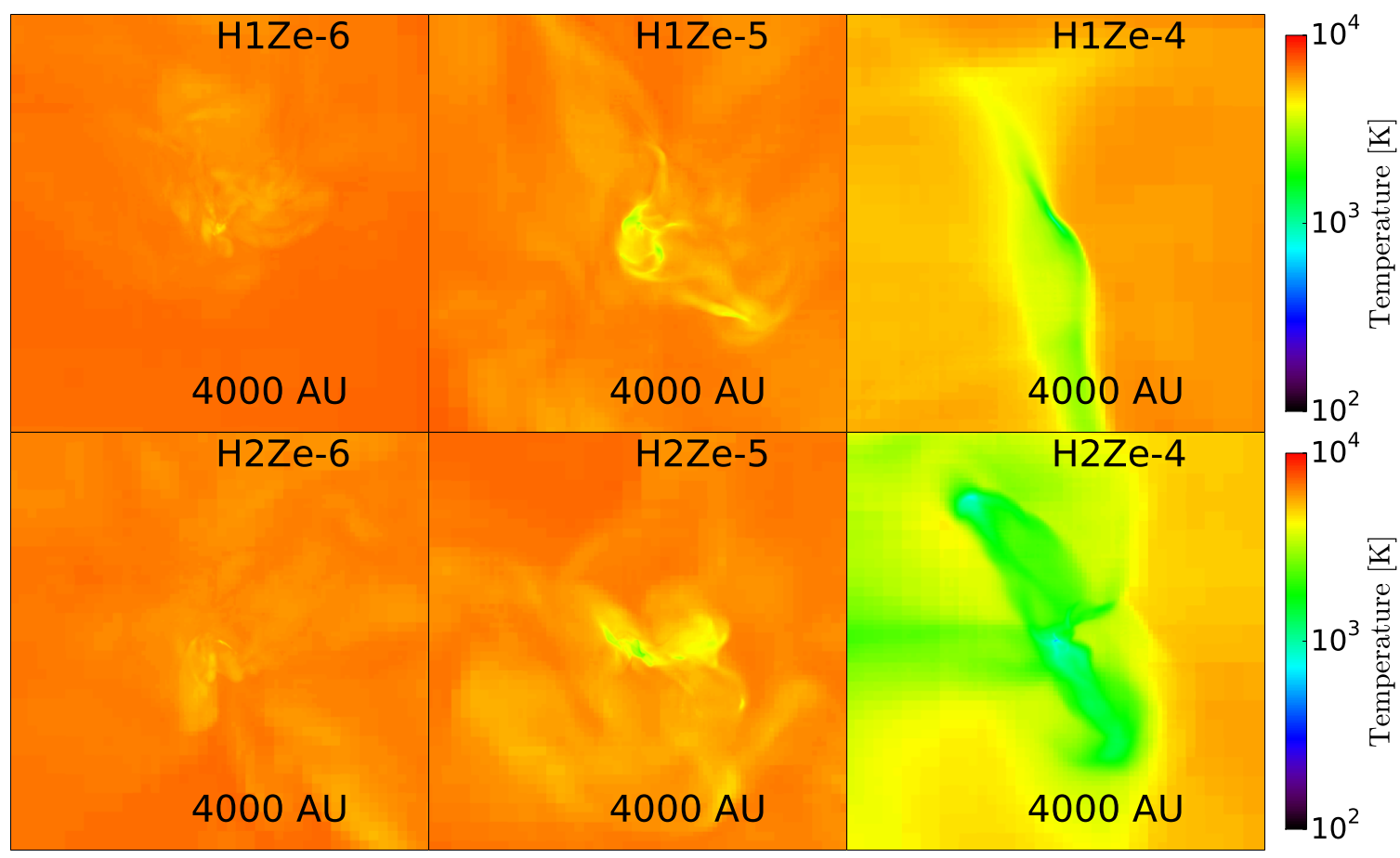

Fig. 7.- Average temperature weighted by the gas density along X-axis for the central $4000 \mathrm{AU}$ of a halo. Each row represents a halo (halo 1 on top and halo 2 on bottom) and each column represent metallicity (increasing from left to right).
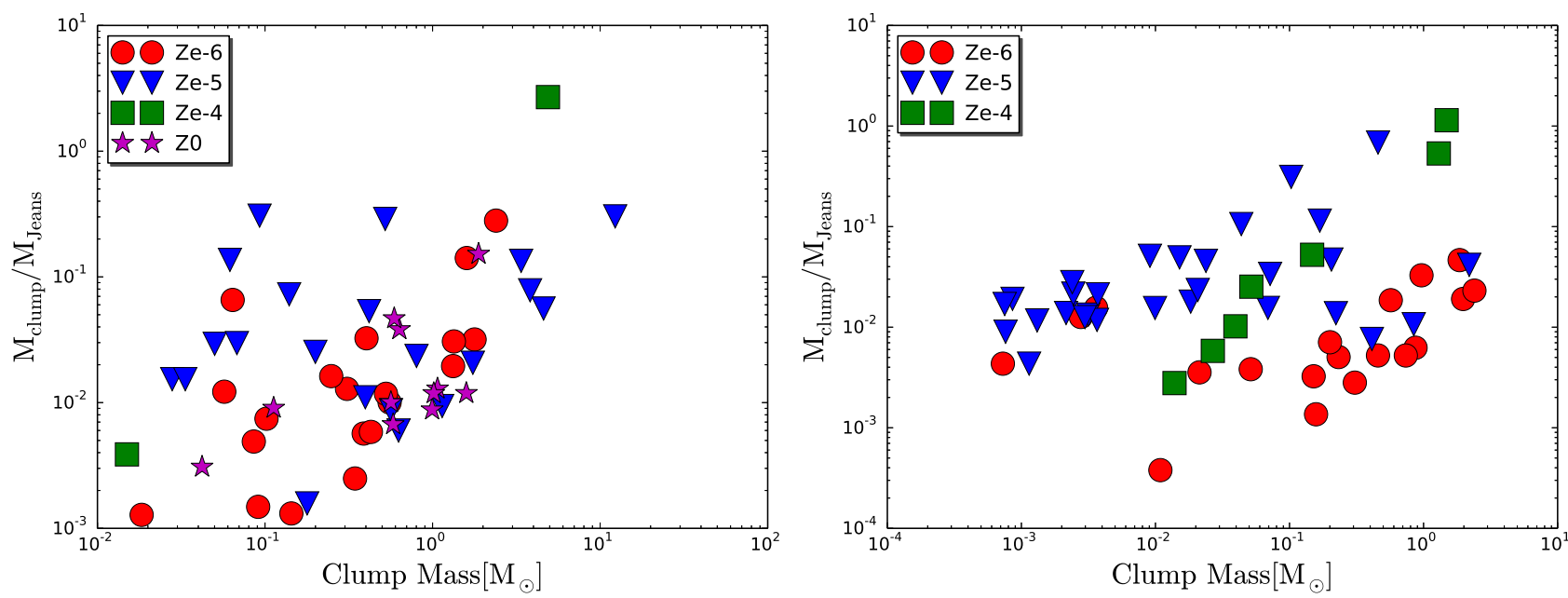

Fig. 8.- The ratio of clump mass to the Jeans mass is plotted against the clump masses for various metallicities. The left panel halo 1 and the right panel halo 2 . The red symbols represent the clumps forming for $Z / Z_{\odot}=10^{-6}$, the blue for $\mathrm{Z} / \mathrm{Z}_{\odot}=10^{-5}$ and the green for $\mathrm{Z} / \mathrm{Z}_{\odot}=10^{-4}$. The clumps for $\mathrm{Z} / \mathrm{Z}_{\odot} \leq 10^{-5}$ are gravitationally unbound while the central clumps for $Z / Z_{\odot}=10^{-4}$ cases are gravitationally bound and more massive. In general, the ratio of clump mass to the Jeans mass increases with clump masses. 


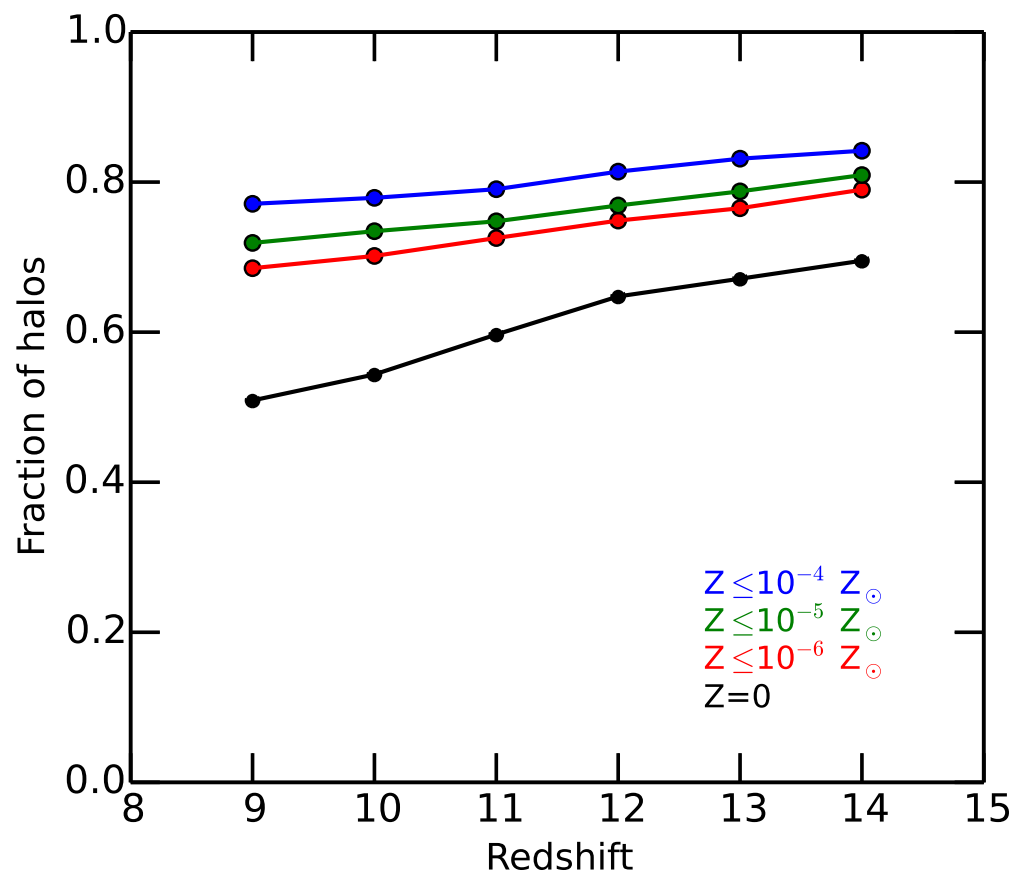

Fig. 9.- Fraction of halos with metallicities below a given value (see legend) as a function of redshift. In this figure, we only show the fraction of halos with masses between $2 \times 10^{7}-10^{8} \mathrm{M}_{\odot}$ in a computational box of size $10 \mathrm{Mpc}$ for $\mathrm{Z} / \mathrm{Z}_{\odot} \leq 10^{-4}$. The fraction of halos with $\mathrm{Z} / \mathrm{Z}_{\odot} \leq 10^{-5}$ is about 1.5 times higher than the metal-free halos. 
—. 2013b, MNRAS, 430, 588

—. 2013c, ApJ, 772, L3

Latif, M. A., Schleicher, D. R. G., Schmidt, W., \& Niemeyer, J. C. 2013d, MNRAS, 436, 2989

Latif, M. A., Schleicher, D. R. G., \& Spaans, M. 2012, A\&A, 540, A101

Latif, M. A., \& Volonteri, M. 2015, MNRAS, 452, 1026

Latif, M. A., Zaroubi, S., \& Spaans, M. 2011, MNRAS, 411, 1659

Loeb, A., \& Rasio, F. A. 1994, ApJ, 432, 52

Lupi, A., Colpi, M., Devecchi, B., Galanti, G., \& Volonteri, M. 2014, MNRAS, 442, 3616

Mortlock, D. J., et al. 2011, Nature, 474, 616

Omukai, K. 2000, ApJ, 534, 809

—. 2001, ApJ, 546, 635

Omukai, K., Schneider, R., \& Haiman, Z. 2008, ApJ, 686, 801

Omukai, K., Tsuribe, T., Schneider, R., \& Ferrara, A. 2005, ApJ, 626, 627

Penston, M. V. 1969, MNRAS, 144, 425

Peters, T., Schleicher, D. R. G., Klessen, R. S., Banerjee, R., Federrath, C., Smith, R. J., \& Sur, S. 2012, ApJ, 760, L28

Portegies Zwart, S. F., Makino, J., McMillan, S. L. W., \& Hut, P. 1999, A\&A, 348, 117

Portegies Zwart, S. F., \& McMillan, S. L. W. 2002, ApJ, 576, 899

Regan, J. A., Johansson, P. H., \& Haehnelt, M. G. 2014a, MNRAS, 439, 1160

Regan, J. A., Johansson, P. H., \& Wise, J. H. 2014b, ApJ, 795, 137

Ritter, J. S., Sluder, A., Safranek-Shrader, C., Milosavljević, M., \& Bromm, V. 2015, MNRAS, 451,1190

Safranek-Shrader, C., Milosavljević, M., \& Bromm, V. 2014, MNRAS, 438, 1669
Sakurai, Y., Hosokawa, T., Yoshida, N., \& Yorke, H. W. 2015, MNRAS, 452, 755

Schleicher, D. R. G., Bovino, S., Latif, M. A., Ferrara, A., \& Grassi, T. 2015, ArXiv e-prints:1504.06296

Schleicher, D. R. G., Palla, F., Ferrara, A., Galli, D., \& Latif, M. 2013, A\&A, 558, A59

Schleicher, D. R. G., Spaans, M., \& Glover, S. C. O. 2010, ApJ, 712, L69

Schneider, R., Ferrara, A., Salvaterra, R., Omukai, K., \& Bromm, V. 2003, Nature, 422, 869

Shang, C., Bryan, G. L., \& Haiman, Z. 2010, MNRAS, 402, 1249

Shlosman, I., Choi, J.-H., Begelman, M. C., \& Nagamine, K. 2016, MNRAS, 456, 500

Smith, B. D., Turk, M. J., Sigurdsson, S., O'Shea, B. W., \& Norman, M. L. 2009, ApJ, 691, 441

Smith, B. D., Wise, J. H., O'Shea, B. W., Norman, M. L., \& Khochfar, S. 2015, MNRAS, 452, 2822

Spaans, M., \& Silk, J. 2006, ApJ, 652, 902

Sugimura, K., Omukai, K., \& Inoue, A. K. 2014, MNRAS, 445,544

Truelove, J. K., Klein, R. I., McKee, C. F., Holliman, II, J. H., Howell, L. H., \& Greenough, J. A. 1997, ApJ, 489, L179+

Tsuribe, T., \& Omukai, K. 2006, ApJ, 642, L61

—. 2008, ApJ, 676, L45

Turk, M. J., Smith, B. D., Oishi, J. S., Skory, S., Skillman, S. W., Abel, T., \& Norman, M. L. 2011, ApJS, 192,9

Tweed, D., Devriendt, J., Blaizot, J., Colombi, S., \& Slyz, A. 2009, A\&A, 506, 647

Van Borm, C., Bovino, S., Latif, M. A., Schleicher, D. R. G., Spaans, M., \& Grassi, T. 2014, A\&A, 572, A22

Venemans, B. P., et al. 2013, ApJ, 779, 24

—. 2015, ArXiv e-prints: 1507.00726

Visbal, E., Haiman, Z., \& Bryan, G. L. 2014, MNRAS, 445,1056 
Volonteri, M. 2010, A\&A Rev., 18, 279

Volonteri, M., \& Bellovary, J. 2012, Reports on Progress in Physics, 75, 124901

Willott, C. J., et al. 2010, AJ, 139, 906

Wise, J. H., Turk, M. J., \& Abel, T. 2008, ApJ, 682, 745

Wise, J. H., Turk, M. J., Norman, M. L., \& Abel, T. 2012, ApJ, 745, 50

Wolcott-Green, J., Haiman, Z., \& Bryan, G. L. 2011, MNRAS, 418, 838

Wu, X.-B., et al. 2015, Nature, 518, 512

Yahil, A. 1983, ApJ, 265, 1047

Yajima, H., \& Khochfar, S. 2015, ArXiv eprints:1507.06701

Yoshida, N., Omukai, K., \& Hernquist, L. 2008, Science, 321, 669

This 2-column preprint was prepared with the AAS $\mathrm{LAT}_{\mathrm{E}} \mathrm{X}$ macros v5.2. 